principle, the fact that a medication with a cardiovascular therapeutic action should prove effective in a cardiovascular pathology with secondary psychiatric symptoms should not surprise anybody; on the contrary, what surprises us is the fact that the authors did not even mention that possibility in the discussion of their case.

Department of Psychiatry

Universidad Autonoma de Madrid

Clinic Puerta de Hierro

San Martin de Porres, 4

28035 Madrid, Spain

Psychogenic amnesia?

SIR: Domb \& Beaman (Journal, March 1991, 158, 423-425) reported the case of "Mr X", whom they consider to be a psychogenic amnesic.

I agree with their assertion that Mr X's loss of personal identity and the circumstances surrounding his admission to hospital suggest a psychogenic amnesia, possibly representing a flight from suicide. There is, however, another rather puzzling aspect to this case, namely, a persisting and selective failure to learn the difficult paired associates of the Wechsler Memory Scale (WMS), despite improvements in performance on other subtests of the WMS. In the experience of our neuropsychological group, this pattern is frequently seen in patients with organic amnesias of bilateral hippocampal origin (Walsh, 1985, 1987). It is also seen in the chronic phase of recovery after left posterior cerebral infarction (Ishikawa et al, 1988), and in patients with left temporal lobe epilepsy in the presence of hippocampal sclerosis (Saling et al, unpublished).

In view of the neuropsychological findings in this case, it would be interesting to know the nature of $\mathrm{Mr}$ $X$ 's two previous suicide attempts. Is it possible that some cases of functional retrograde amnesia represent massive psychological elaboration around a kernel of organic involvement?

IshikAWA, K., DARBY, D. G., SAlING, M. M. et al (1988) Verbal and visual recognition memory improvement in left posterior cerebral artery infarction with general amnesic syndrome. In Proceedings of the Thirteenth Annual Brain Impairment Conference (eds $\mathbf{M}$. Matheson \& H. Newman), pp. 128-162. Sydney.

WALSH, K. W. (1985) Understanding Brain Damage: A Primer of Neuropsychological Evaluation. Edinburgh: Churchill Livingstone.

(1987) Neuropsychology: A Clinical Approach. Edinburgh:

Churchill Livingstone.

Michael M. Saling

The University of Melbourne

Parkville 3052

Australia

\section{Clinical interviews with children and adolescents}

SIR: It is not customary for authors to write to editors commenting upon reviews of their books and indeed until now I have never done so. However I feel that some comment is needed about Dr Dora Black's review of Clinical Interviews with Children and Adolescents.

Dr Black says that "the author seems never to have got clear in his mind what his aims are". I believe that the aims of the book were stated clearly in the introduction. Re-reading this confirms my belief.

She goes on to say that I seem "to be considering therapeutic interviews as well as diagnostic ones" but that I miss them off the list of reasons for interviewing children. This statement is just wrong. Treatment interviews, defined as aiming to promote rather than impose change, are clearly listed on page 12 . Dr Black seems to have missed the point I make, also on page 12 , contrasting 'manipulative' interviews which attempt to impose change - with 'treatment' interviews, which aim to promote it.

Earlier in the review Dr Black says that the idea of my book "is an original one". This is not the case. A book entitled Interviewing Children and Adolescents was published by John Rich in 1968 and is cited by me. Indeed, the classification of interviews upon which Dr Black comments is derived, with acknowledgement, from Rich's book.

I find it difficult to avoid the conclusion that Dr Black's review was written after only a cursory look through the book.

Department of Psychiatry

PHILIP BARKer

Alberta Children's Hospital

1820 Richmond Road S.W.

Calgary, Alberta

Canada T2T $5 C 7$

AUTHOR'S REPLY: I certainly read Professor Barker's book from cover to cover. Our differences hinge on the fact that the whole book is devoted to what Rich called fact-finding interviews, and the author states that "psychotherapy techniques themselves fall outside the scope of this book" (p. 12) yet in the chapter on "Termination" there is advice about resolving attachment to the therapist. It is this that led me to state that the aims were not clear. Perhaps it would have been better to say that the aims were stated but a trainee would not emerge from reading the book with a clear idea of whether the interviews described were for assessment or therapy. As I said originally, the book is of value as the distillation of the experience of a sympathetic and skilled 